\title{
The Derived Transfer and Reversal of Mood Functions Through Equivalence Relations: II
}

Article in The Psychological record · June 2007

DOI: $10.1007 / \mathrm{BF} 03395583 \cdot$ Source: OAI

CITATIONS

14

6 authors, including:

Yvonne Barnes-Holmes

Ghent University

169 PUBLICATIONS 6,423 CITATIONS

SEE PROFILE

Carmen Luciano

Universidad de Almería

176 PUBLICATIONS 3,571 CITATIONS

SEE PROFILE
READS

114

Miguel Rodríguez-Valverde

Universidad de Jaén

29 PUBLICATIONS 875 CITATIONS

SEE PROFILE

Some of the authors of this publication are also working on these related projects:

Developing and testing Acceptance and Commitment Therapy protocols focused on disrupting repetitive negative thinking for the treatment of emotional disorders View project

Experimental analysis of Acceptance and Commitment Therapy (ACT) strategies and application of a brief protocol in patterns of psychological inflexibility View project 


\title{
THE DERIVED TRANSFER AND REVERSAL OF MOOD FUNCTIONS THROUGH EQUIVALENCE RELATIONS: ॥
}

\author{
JANE CAHILL, YVONNE BARNES-HOLMES, \\ and DERMOT BARNES-HOLMES \\ National University of Ireland, Maynooth
}

\begin{abstract}
MIGUEL RODRIGUEZ-VALVERDE and CARMEN LUCIANO University of Almería, Spain
\end{abstract}

\author{
PAUL M. SMEETS \\ Leiden University, Netherlands
}

\begin{abstract}
Recent research has demonstrated the transfer of induced mood functions through equivalence relations by means of a musical mood-induction procedure. The research described in this article replicated and extended such work, primarily with the inclusion of a baseline and two types of reversal procedures. First, 16 adult participants were trained and tested for the formation of two three-member equivalence classes (A1-B1-C1 and A2-B2-C2). A musical mood-induction procedure was used to induce happy and sad mood states in the presence of the B stimuli, and a transfer of mood functions test was administered to determine whether the corresponding mood functions had transferred via equivalence to the $\mathrm{C}$ stimuli. Participants were then exposed to either Reversal 1, in which only the functions attached to the B stimuli were reversed, or to Reversal 2, in which the structure of the equivalence classes was altered. At all stages throughout the experiment, participants were asked to rate their current mood state by using mood and incentive rating scales. The findings of the current study replicated the results of previous research in demonstrating a clear transfer of mood functions via equivalence relations. This outcome is compared with the existing literature about mood induction and the transfer of mood functions.
\end{abstract}

Stimulus equivalence has attracted considerable empirical and theoretical interest among behavioral researchers in the 3 decades since Sidman's seminal study in 1971. In a typical equivalence study, a participant might be trained to select comparison stimulus $B$ and comparison stimulus $C$ in the presence of sample stimulus $A$ (thus explicitly establishing A-B and $A-C$ relations). Subsequently, the participant may match $B$ to $A$ and $C$ to $A$ (symmetry relations), as well as $B$ to $C$ and $C$ to $B$ (combined symmetry and transitivity or equivalence relations) without further training.

Address correspondence to Yvonne Barnes-Holmes, Department of Psychology, National University of Ireland, Maynooth, Co. Kildare, Ireland. (E-mail: Yvonne.BarnesHolmes@ nuim.ie). 
When such novel performances emerge, the stimuli (e.g., A, B, and C) are said to participate in an equivalence class (A-B-C), and a host of studies has demonstrated the derivation of these types of relations (see Hayes, Barnes-Holmes, \& Roche, 2001, and Sidman, 1994, for reviews).

A phenomenon that is closely related to the concept of equivalence is the derived transfer of function effect. For illustrative purposes, imagine that three stimuli, A, B, and C, participate in an equivalence class and a reinforcing function is attached to $A$. Subsequently, both the $B$ and the $C$ stimuli may acquire the reinforcing function that was directly established for $A$, even though the function was never directly attached to these latter stimuli. It has been argued that the concept of the transfer of function is important, because it is this behavioral property that provides stimulus relations with psychological content (Hayes et al., 2001). Many studies have demonstrated transfers of a range of stimulus functions, including self-discrimination and sexual arousal functions (Dymond \& Barnes, 1994; Roche \& Barnes, 1997). Furthermore, some researchers have highlighted the potential clinical implications of these effects. For example, Dougher, Auguston, Markham, Greenway, and Wulfert (1994) reported the transfer of differential shock functions through equivalence relations with the use of skin conductance responses as a measure of autonomic arousal. This work highlighted the possible utility of the transfer of function effect as a behavioral process by which individuals may develop fear or avoidance, or both, of arbitrary stimuli that have never been directly paired with aversive events.

Another domain in which the derived transfer of functions may be relevant to the understanding of clinical phenomena was highlighted in a recent study that focused on the mood-generating functions of particular stimuli. Barnes-Holmes, Barnes-Holmes, Smeets, and Luciano (2004) reported a transfer of derived mood functions through equivalence relations with the use of a musical mood-induction procedure. In Phase 1 of the study, participants were exposed to conditional discrimination training on four matching-to-sample tasks (i.e., A1-B1, A2-B2, A1-C1, and $\mathrm{A} 2-\mathrm{C}_{2}$ ) and underwent subsequent testing in Phase 2 for the formation of two equivalence classes (e.g., A1-B1-C1 and A2-B2-C2). After the establishment of the designated equivalence classes was demonstrated, a musical mood-induction technique was employed in Phase 3 to induce a happy mood in the presence of one of the B stimuli and a sad mood in the presence of the other B stimulus (e.g., B1 = happy mood and B2 = sad mood). In Phase 4, participants were tested for the emergence of these mood functions in the presence of the B stimuli, and in Phase 5 they were tested for the transfer of mood functions through the equivalence relations to the C stimuli (e.g., C1 = happy mood and C2 = sad mood).

The self-report mood and incentive rating measures used in the study performed by Barnes-Holmes et al. (2004) indicated that the majority of participants showed the derived transfer of the appropriate happy and sad mood functions through the equivalence relations. In effect, participants produced high mood and incentive ratings in the presence of the $\mathrm{B}$ and 
C stimuli that had been directly and indirectly related to happy music, respectively, and low ratings in the presence of the $B$ and $C$ stimuli that had been directly and indirectly related to sad music, respectively. These findings provided the first demonstration of the transfer of mood functions and supported previous research on the transfer of psychological functions through derived relations (Barnes \& Keenan, 1993; Barnes et al., 1995; Dougher et al., 1994). In line with Dougher et al., the researchers highlighted the clinical implications of this effect and argued that the derived transfer of mood functions may provide a means of understanding alterations in mood states, or mood swings, that occur in the presence of stimuli or events that have not previously been directly associated with any specific emotional event.

The current work was an attempt to replicate the study by BarnesHolmes et al. (2004) and to address a number of weaknesses that could have reduced the importance and applicability of their results. First, the original study lacked a baseline mood functions test, and thus it is possible that the experimental stimuli possessed unknown and possibly contaminating preexperimentally established mood functions. The taking of a baseline measure in the current study should provide strong evidence that the induced and derived mood changes are a direct result of the experimental contingencies. Second, the original study also lacked a reversal procedure, and thus the research did not show that the transfer effects, once established, were manipulable within the experimental setting. The current study, therefore, employed two different types of reversal procedure with the derived transfer of mood effect. In Reversal 1 , the functions attached to the B stimuli were simply reversed, while the two equivalence classes trained and tested previously remained the same. In Reversal 2, the structure of the equivalence classes was altered by switching the contingencies for $A-B$ responding, while the functions attached during the mood functions training remained the same. The current study thus aimed to replicate the work of Barnes-Holmes et al. and to demonstrate that the original findings were unlikely owing to uncontrolled preexperimental functions and that derived transfer effects are reversible within the experimental context. Demonstrating these effects, particularly the within-experimental reversibility of derived mood functions, will further bolster the relevance of the research to a behavior analytic interpretation of human mood disorders.

\section{Participants}

Method

Sixteen participants, 8 female and 8 male, with ages ranging from 18 to 28 (mean, 23) years, participated in the current study. All participants were recruited through public announcements at the National University of Ireland, Maynooth, and were either graduate or undergraduate students. None of the participants had previously undertaken courses in psychology, nor did they receive any remuneration for their participation. 


\section{Setting}

The experiment was conducted in a quiet room in an experimental psychology laboratory at the National University of Ireland, Maynooth. During all training and testing phases, both the experimenter and the participant were seated beside one another at an experimental table. During all occasions in which mood ratings were conducted, the experimenter was seated on a third chair at the back of the room and to the rear of the participant. All participants took part in the experiment individually. During experimental sessions, participants were asked not to leave the room, and none did so. The study consisted of three experimental stages. For all participants the maximal length of time spent participating in the experiment was $100 \mathrm{~min}$.

\section{Materials}

A stop clock was used to record the stimulus exposure times and the delays between the exposures. Drinking water and reading materials (i.e., magazines and newspapers) were also available in the experimental room for use during predetermined rest periods.

Stimulus cards. Twenty-eight stimulus cards were used throughout the study. Each card consisted of a piece of white laminated A4 paper. Twentyfour of the cards depicted three stimuli, while the remaining four cards depicted only one stimulus. All of the stimuli were three-letter nonsense syllables: ZID, YIM, VEK, ZOM, DAX, and BEH. For convenience, each of the six nonsense syllables was designated with an alphanumeric label: A1 (ZID), A2 (YIM), B1 (VEK), B2 (ZOM), C1 (DAX), and C2 (BEH). Participants were never made aware of these labels. All nonsense syllables were printed in black bold ink, 48-point Arial font. Each card depicting three nonsense syllables contained a sample stimulus presented in the center top portion of the card, with two comparison stimuli positioned to the left and to the right below the sample. On the four cards depicting only one stimulus, the sample was in the center of the card.

Musical mood-induction materials. The current study involved two excerpts of classical music that were used for the induction of happy and sad mood states. These materials were identical to those employed in the original study by Barnes-Holmes et al. (2004). The efficacy of these particular musical excerpts for this type of mood induction had also been demonstrated by Samson and Rachman (1989) and by Clark and Teasdale (1985), who reported the induction of appropriate mood states in $94 \%$ and $87 \%$ of participants, respectively. The excerpt of classical music used to induce a happy mood was Mozart's "Divertimento 136," and the excerpt used to induce a sad mood was Albinoni's Adagio in G Minor. Each musical excerpt was downloaded from the Internet in the form of an MP3 file that was converted into a standard audio format and recorded onto a CD for use in a standard CD player. Although both excerpts in the original MP3 form were approximately 7 min long, each was digitally compressed so that both were of exactly the same length (420 s). During the appropriate experimental phases, the music was presented to each 
participant with the use of standard headphones and a CD player. Prior to commencing the experiment, each participant was presented with a musical excerpt not used in the experiment and was asked to use the headphones to select the level of volume that was clearly audible and most comfortable.

Self-report rating measures. Two types of self-report rating measures were employed in the current study: a mood rating scale and an incentive rating scale, both of which were identical to those employed by BarnesHolmes et al. (2004). The mood rating scale was adopted from a study by Brown and Mankowski (1993), in which significant differences in reported mood states were observed after positive and negative mood inductions. The current mood scale consisted of six identifiable mood states: three negative (i.e., depressed, low, and unhappy) and three positive (i.e., elated, glad, and joyful). Participants were required to indicate, on a 5point scale, the extent to which they were currently experiencing each mood, where 1 denoted "Not at all" and 5 denoted "Extremely." The mood scale contained a brief instruction at the top of the sheet as follows: "Please indicate on each of the scales below the extent to which you are experiencing each mood at this moment."

The incentive rating scale had been adapted from a study by Clark (1983), in which the sensitivity of incentive ratings to the induction of different mood states was demonstrated (see also Clark \& Teasdale, 1985; Teasdale \& Spencer, 1982). The incentive scale consisted of six potentially pleasurable activities: having coffee with old friends; curling up with a good book; taking a long, warm bath; taking some physical exercise alone; going for a meal with some new and interesting people; and listening to your choice of music with a group of friends. Participants were required to rate, on an 11-point scale, how much they would like to engage, given the opportunity at the moment of rating, in each of six potentially pleasurable activities, where 1 denoted "Not at all" and 11 denoted "Very, Very Much." The incentive scale contained a brief instruction at the top of the sheet as follows: "Please indicate on the scales below the extent to which you would like to engage in each of the following pleasurable activities at this moment."

\section{Experimental Sequence}

The current study consisted of Stages 1,2, and 3, conducted in that order. A schematic representation of the stages and component phases is presented in Figure 1. Stage 1 constituted a baseline mood rating stage in which the participants were asked to produce mood and incentive ratings in the presence of some of the experimental stimuli that would subsequently be involved in the mood-induction and mood-transfer procedures. Stage 2 consisted of the equivalence training and testing and the mood-induction procedures. This stage contained five experimental phases. Phase 1 consisted of conditional discrimination training (A1-B1, A2-B2, A1-C1, and A2-C2 relations) in a standard matching-to-sample format, followed in Phase 2 by symmetry probes and equivalence probes 


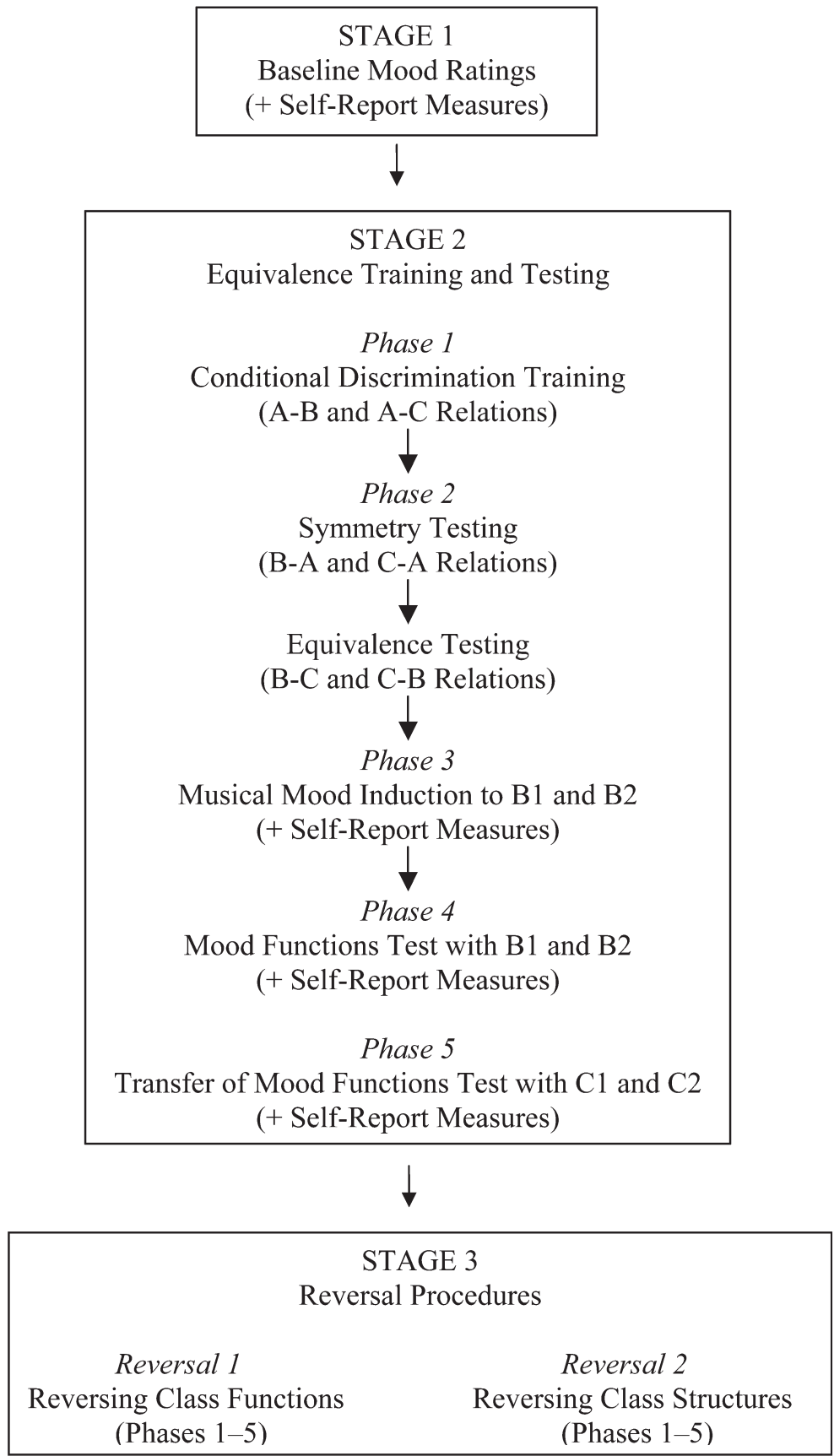

Figure 1. Schematic representation of the three experimental stages and their component phases (where appropriate). 
for the formation of two three-member equivalence classes (A1-B1-C1 and A2-B2-C2). In Phase 3, a musical mood-induction procedure was employed to establish "happy" and "sad" mood functions in the presence of the B stimuli (e.g., B1-happy and B2-sad), and in Phase 4, participants were exposed to a test to determine whether the appropriate functions had become attached to the $B$ stimuli. In Phase 5, they received a transfer of functions test to determine whether the appropriate mood functions had transferred from the $B$ stimuli to the $C$ stimuli via the equivalence relations (e.g., C1-happy and C2-sad). Stage 3 consisted of the same five experimental phases as were in Stage 2, except that two types of reversal procedure were compared: one in which the structure of the equivalence classes was reversed (Reversal 1) and the other in which the functions of the equivalence classes were reversed (Reversal 2).

\section{Programmed Consequences}

A correct response during all training trials was followed by verbal feedback with the words "Yes, that's correct." An incorrect response was always followed by the verbal feedback "No, that's incorrect." Programmed consequences were employed with all training trials conducted in Phase 1 of Stages 2 and 3, but no programmed consequences followed any test trial or other type of experimental trial.

\section{Procedure}

Stage 1: Baseline stimulus rating stage.

This stage involved one presentation of each of the four stimulus cards that contained only one nonsense syllable (i.e., B1, B2, C1, and C2). After the first card was placed on the table, the participant was provided with the following instruction: "Please concentrate on this item for the next $60 \mathrm{~s}$, after which I will ask you to fill out two brief rating measures." For the $60 \mathrm{~s}$, during which the card remained on the table, the experimenter was seated behind the participant and to the right. When the $60 \mathrm{~s}$ had passed, the card was removed and the participant was presented with the two self-report rating measures (one mood and one incentive). After the rating measures were completed for the first stimulus, the other three stimuli and the pairs of ratings that followed each stimulus were presented in the same way. The order in which the four stimuli were presented was randomized across participants.

\section{Stage 2: Training and testing in equivalence and mood transfer.}

Stage 2 consisted of five phases of training and testing (see Figure 1) that were identical to those employed by Barnes-Holmes et al. (2004).

Phase 1: Conditional discrimination training. Participants were trained to establish two A-B (A1-B1, A2-B2) and two A-C (A1-C1 and A2-C2) relations. The $A-B$ relations were always presented first, and participants could proceed to $A-C$ training only when they had reached the criterion on the A-B relations. Prior to the first A-B training trial, the experimenter read aloud the following instructions:

In this phase of the experiment, you will be shown a number of 
cards. Each has three items on it, one at the top, along with two items below it. Your task is to learn as quickly as possible through trial and error to pick the one from the bottom that goes with the top item. I will tell you when you are correct more frequently at the start than later on.

All of the training and testing conducted within the current study was composed of blocks of eight randomized trials. Each block of eight A-B trials contained four exposures to the A1-B1 and A2-B2 trial types. When $A 1$ was the sample, reinforcement was provided for selecting $B 1$, and when A2 was the sample, reinforcement was provided for selecting B2. The left-right locations of the comparison stimuli alternated randomly across all matching-to-sample trials throughout the experiment. To achieve the training criterion, participants were required to produce eight consecutively correct responses on the A-B relations. Participants were then exposed to training on the $A-C$ relations (A1-C1 and $A 2-C 2)$, which was identical to the $A-B$ training, except that the $C$ stimuli were now employed as comparisons. Once again, participants were required to achieve eight consecutively correct responses before proceeding to mixed $A-B$ and $A-C$ training. During the mixed training, participants received two exposures to each of the four $A-B$ and $A-C$ trial types. Once again, participants were required to reach the mastery criterion of eight consecutively correct responses before proceeding to Phase 2 .

Phase 2: Symmetry and equivalence tests. Participants were first exposed to four trial types that tested for the emergence of symmetry. These trial types involved the symmetrical relations of B1-A1, B2-A2, $\mathrm{C} 1-\mathrm{A} 1$, and C2-A2. The symmetry test consisted of blocks of eight randomized trials. To pass the symmetry test, participants were required to produce eight consecutively correct responses.

Participants who passed the symmetry test proceeded immediately to the equivalence test trials. Participants who did not pass the symmetry test on the first block of eight trials were reexposed immediately to the mixed $A-B$ and $A-C$ training. If participants produced eight consecutively correct responses across the first eight training trials, they proceeded once again to the symmetry test. If, however, they did not reach this training criterion, they were returned to the beginning of Stage 2 and proceeded once again through $A-B, A-C$, and mixed training before reexposure to the symmetry test. This pattern of retraining and symmetry testing continued until all participants had passed the symmetry test. Immediately thereafter, participants were exposed to the equivalence test trials.

The equivalence test was conducted in exactly the same manner as the symmetry test, except that $\mathrm{B}-\mathrm{C}$ and $\mathrm{C}-\mathrm{B}$ relations (i.e., $\mathrm{B} 1-\mathrm{C} 1$, $\mathrm{B} 2-\mathrm{C} 2, \mathrm{C} 1-\mathrm{B} 1$, and $\mathrm{C} 2-\mathrm{B} 2)$ were probed. If a participant produced eight consecutively correct responses on the first block of eight equivalence trials, it was assumed that two three-member equivalence classes, A1-B1-C1 and A2-B2-C2, had emerged. If participants failed to show equivalence responding, they were reexposed to the mixed A-B and A$C$ training and then to the symmetry test before being reexposed to the 
equivalence test probes. This cycle of retraining and retesting continued until each participant demonstrated equivalence responding.

Phase 3: Mood functions training. In Phase 3, the musical moodinduction procedure was used to establish happy and sad mood states in the presence of the B stimuli. The mood functions established for B1 and B2 were counterbalanced across participants. Consider, for example, the induction of a happy mood in the presence of B1. In this case the experimenter presented the card containing B1 and then provided the following instructions:

In this phase of the experiment, you will be asked to listen to a piece of classical music. It is important that you concentrate on the item on the card in front of you, while you are listening to the music. This section of music should help you to develop a happy mood. However, music alone cannot create the desired mood, so you should try to think about something that makes you happy. You may find it especially useful to concentrate on happy events that you have personally experienced.

After receiving the instructions, the participant was provided with a set of headphones attached to the CD player. The happy music, "Divertimento 136," by Mozart was played through the headphones for $7 \mathrm{~min}$. During this time, the participant was required to focus on the card containing the B1 stimulus. Once the $7 \mathrm{~min}$ had passed, the musical excerpt ended and the B1 stimulus card was removed. The participant was then allowed a 2-min break before the B2 stimulus was presented.

For the induction of a sad mood, the experimenter presented the relevant B stimulus (e.g., B2) and provided the following instructions:

This time, you will be asked again to listen to a piece of classical music and to concentrate on the item on the card in front of you. This section of music should help you to develop a sad mood. However, music alone cannot create the desired mood, so you should try to think about something that makes you sad. You may find it especially useful to concentrate on sad events that you have personally experienced.

The music of Albinoni's Adagio in $G$ minor was played through the headphones for $7 \mathrm{~min}$. while the $\mathrm{B}$ stimulus card remained on the table in front of the participant. The participant was then allowed another 2-min break before proceeding to the next phase.

Phase 4: Mood functions test. This phase involved a test to determine whether the happy and sad mood functions had been established appropriately for each B stimulus (e.g., B1-happy and B2-sad). Once again, the order in which the $B$ stimuli were presented in Phase 4 was counterbalanced across participants. When the participant received the first B stimulus, the following instruction was provided: "Please concentrate on this item for the next $60 \mathrm{~s}$, after which I will ask you to fill out two brief rating measures." When the $60 \mathrm{~s}$ had passed, the $B$ stimulus was removed 
and the participant was presented with one set of incentive and mood rating measures. After both rating measures were obtained, the participant was given a 5-min break during which she or he was allowed access to the drinks and reading materials that were available in the experimental room.

Immediately after the 5-min break, the second B stimulus, followed by two rating measures, was presented in exactly the same manner as before. The appropriate happy and sad mood functions were deemed to have been established for the $B$ stimuli if higher emotional ratings were reported for the stimulus that had been paired with the happy music than were reported for the stimulus paired with the sad music. Another 5-min break was scheduled for participants before proceeding to Phase 5 .

Phase 5: Transfer of mood functions test. During this phase of the experiment, participants were exposed to a test for the derived transfer of the happy and sad mood functions from B1 and B2 to C1 and C2, respectively. This phase of the experiment was identical to Phase 4, except that the $\mathrm{C}$ stimuli (rather than the B stimuli) were presented and relevant ratings were obtained. The derived transfer of happy and sad mood functions was defined as the emergence of similar mood and incentive ratings for the two $\mathrm{C}$ stimuli as for the two $\mathrm{B}$ stimuli that were related via equivalence (e.g., B1/high mood rating $\rightarrow \mathrm{C} 1 /$ high mood rating and $\mathrm{B} 2 /$ low mood rating $\rightarrow \mathrm{C} 2 /$ low mood rating).

Stage 3: Reversals of the structure or function of the equivalence classes and reversal of mood transfer.

Stage 3 involved one of two reversal procedures (i.e., Reversal 1 and Reversal 2). Eight participants were exposed to Reversal 1, while the other 8 participants were exposed to Reversal 2.

Reversal 1. Reversal 1 consisted of a simple procedure in which the mood functions attached to the B stimuli in Phase 3 of Stage 2 were reversed. In effect, Reversal 1 consisted of five experimental phases that were identical to Stage 2, except that alternate mood functions were established for the B stimuli. In other words, the relations targeted during both the conditional discrimination training and the equivalence testing were identical to the previous stage for these participants. Once participants had completed all five phases of Reversal 1, their participation was complete.

Reversal 2. The participants were reexposed to all five phases of Stage 2, but $\mathrm{A} 1-\mathrm{B} 1$ and $\mathrm{A} 2-\mathrm{B} 2$ conditional discriminations were reversed (i.e., $\mathrm{A} 1-\mathrm{B} 2$ and $\mathrm{A} 2-\mathrm{B} 1$ relations). This reversal of the conditional discriminations meant that the target equivalence relations for these participants also changed (B2-C1 and B1-C2). Apart from this reversal, all of the stimulus arrangements were identical to those employed previously and the same mood functions were attached to same B stimuli. Completion of all five phases of Reversal 2 in Stage 3 marked the end of the experiment.

\section{Interobserver Reliability}

Thirty-five percent of training and test trials in Stages 2 and 3 were observed by an independent rater who had no knowledge of experimental 
psychology. The observer was behind the participant and to the left. The observer could not see the experimenter's data sheet during the experimental sessions. Agreement between the observer's and the experimenter's recordings was $100 \%$.

\section{Results}

All of the stimuli presented during the baseline were neutral but would subsequently have putative happy and sad mood functions attached to them. Hereafter the stimuli will be referred to as "happy B," "happy C," "sad B," and "sad C." For the initial analyses the data from both types of reversal procedures were collapsed (reversed B functions or reversed A$B$ relations). For all aspects of the results, the data obtained with the mood and incentive rating scales were analyzed separately.

\section{Mood Rating Scores}

The raw data obtained from participants' responses to the mood scale were manipulated for the purposes of scoring. That is, because the mood rating measure consisted of three happy and three sad mood items, and scores were provided on the same 5-point scale, the scores for the three negative items were reversed. For example, if a participant reported that she felt "extremely" depressed, low, or unhappy, these responses were each recorded by the participant as 5 but were then each rated by the experimenter as 1 . Each time a participant completed a mood rating scale, the total rating across the six mood items was calculated and a mean mood performance for that rating scale on that occasion was calculated for each participant. The mean mood ratings and standard errors obtained for each of the three experimental stages are presented in Figure 2.

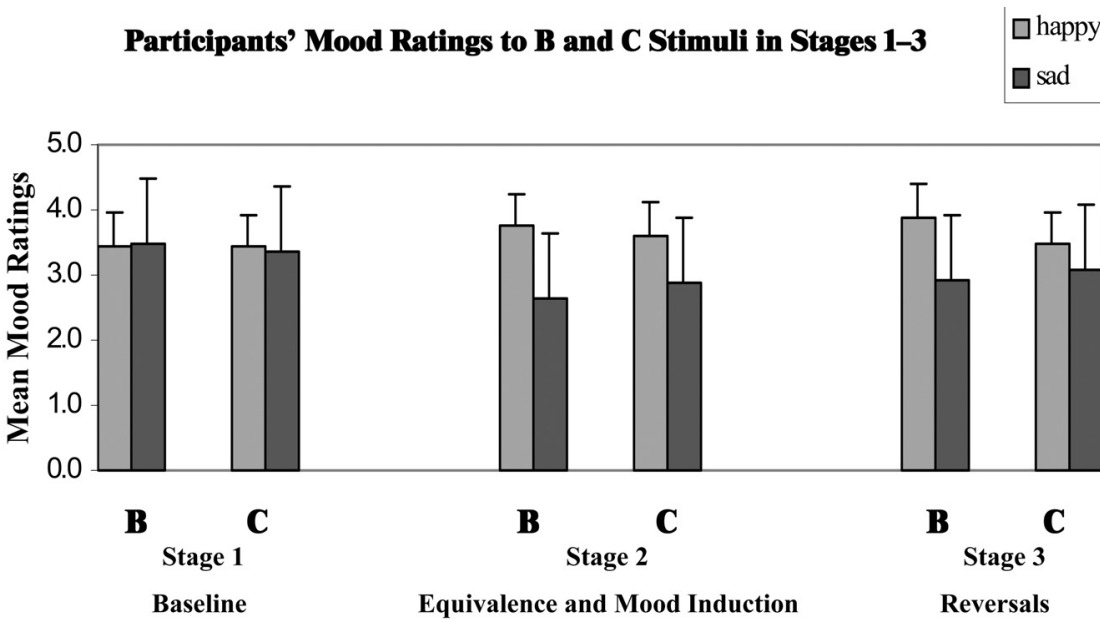

Figure 2. Mean mood ratings and standard error bars for the $B$ and $C$ stimuli in Stages 1, 2, and 3 . For the Stage 3 reversals, the functions of the two $B$ stimuli were reversed for half of the participants (Reversal 1) and the A-B conditional discriminations were reversed for the remaining participants (Reversal 2). 
Almost no differences were observed between B1 and B2 or between $\mathrm{C} 1$ and $\mathrm{C} 2$ during Stage 1 prior to the experimental manipulations. In contrast, the mood ratings recorded for the happy and sad B stimuli in Stage 2 differed considerably (3.75 and 2.65, respectively). Furthermore, these differences appeared to transfer to the happy and sad $C$ stimuli (3.60 and 2.89, respectively). A similar pattern of differences was also observed in the mood data recorded after the reversal procedures in Stage 3 (happy and sad B were 3.88 and 2.91, respectively, and happy and sad $C$ were 3.47 and 3.09 , respectively).

A $3 \times 2 \times 2$ repeated-measures analysis of variance (ANOVA) was conducted with stage (Stages 1,2, and 3), stimulus function (happy and sad), and stimulus type ( $B$ and $C$ ) as within-participant variables. The results of this analysis indicated a significant main effect for stimulus function $(F[1,15]=15.569, p=.0013)$ but not for stimulus type or stage. A significant interaction between stage and stimulus function $(F[2,30]$ $=9.231, p=.0008$ ) was also obtained, supporting the prediction that the difference between the happy and sad stimuli would emerge only in Stages 2 and 3 (i.e., after the mood-induction procedures).

Given the significant interaction effect, three separate $2 \times 2$ ANOVAs were conducted for each exposure. (A Bonferroni correction $[p<.01]$ was employed to control for Type 2 error.) The ANOVA for Stage 1 indicated no significant main effect for stimulus function $(p=.9526)$ or stimulus type $(p=.4831)$ and no interaction effect. The ANOVA for Stage 2 revealed a significant effect for stimulus function $(F[1,15]=14.194, p=.0019)$ but not for stimulus type and with no interaction effect. The same pattern was obtained for the Stage 3 ANOVA with a significant effect for stimulus function $(F[1,15]=12.785, p=.0028)$ but not for stimulus type or for the interaction. The statistical analyses thus supported the conclusion that the mood-induction procedure, combined with the equivalence training and testing, was successful in establishing differential mood functions for the $\mathrm{B}$ and $\mathrm{C}$ stimuli during Stages 2 and 3.

\section{Incentive Rating Scores}

Unlike the mood scores, the incentive ratings did not need to be reversed for scoring. A high incentive score was taken to indicate a happy mood, whereas a low incentive score was taken to indicate a sad mood. The mean incentive ratings and standard errors obtained for each of the three experimental stages are presented in Figure 3. Very little difference was recorded between the two B stimuli at baseline; the difference between the two $\mathrm{C}$ stimuli, although slightly larger, was in the wrong direction. After the mood-induction procedures, the predicted differences between the happy and sad stimuli emerged, although they remained relatively small (all of the mean incentive ratings being between 6 and 7 points). A $3 \times 2 \times 2$ repeated-measures ANOVA showed nonsignificant main effects for stage $(p=.5240)$, stimulus function $(p=.2144)$, and stimulus type $(p=.3769)$, and no interaction effects at the $p<.05$ level. Consistent with the previous findings of Barnes-Holmes et al. (2004), 
Participants' Incentive Ratings to B and C Stimuli in Stages 1-3
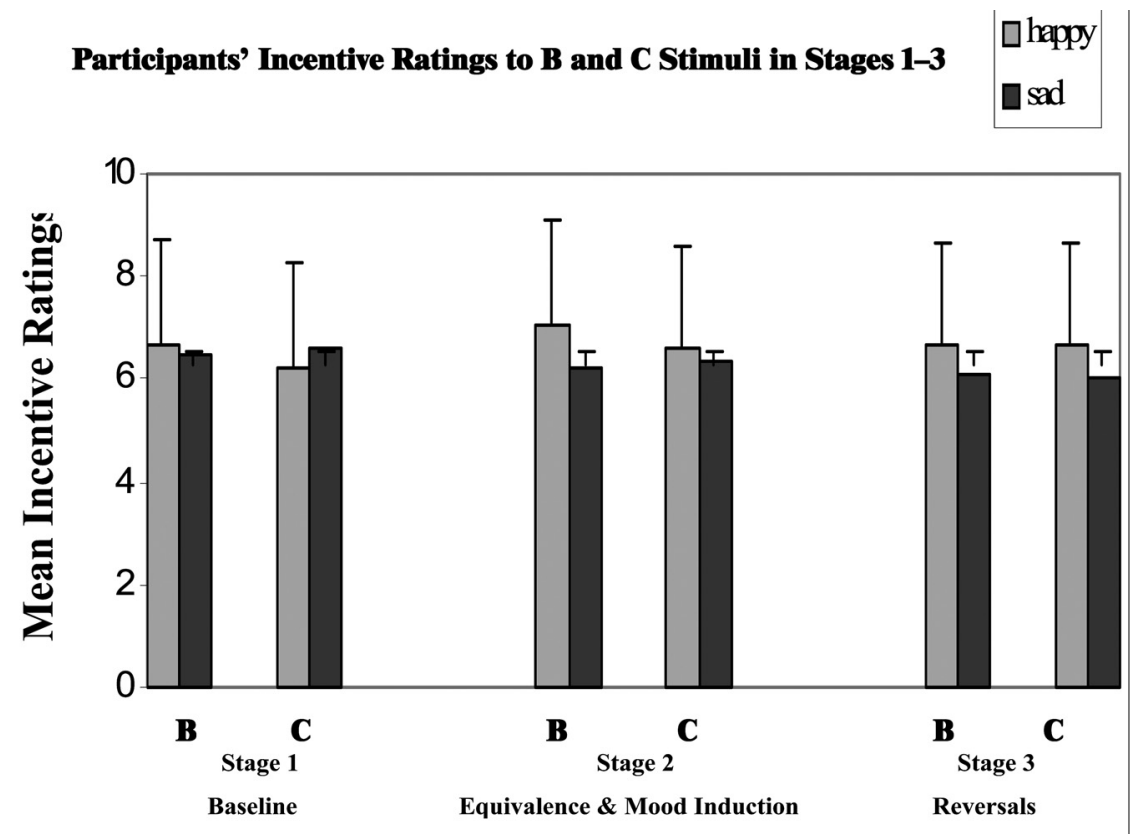

Figure 3. Mean incentive ratings and standard error bars for the $\mathrm{B}$ and $\mathrm{C}$ stimuli in Stages 1, 2, and 3. For the Stage 3 reversals, the functions of the two B stimuli were reversed for half of the participants (Reversal 1) and the A-B conditional discriminations were reversed for the remaining participants (Reversal 2).

the incentive measures, although in the predicted direction, did not yield statistically significant effects.

\section{Comparing Reversals 1 and 2}

To determine whether the two types of reversal procedure produced different effects, two individual mixed $2 \times 2 \times 3$ repeated-measures ANOVAs were conducted (one for mood and the other for incentive). The mood analyses revealed no interaction effects and nonsignificant effects for type of reversal, stimulus type, and stage. Similarly, the incentive analyses revealed nonsignificant effects for reversal, stimulus type, and stage, with no interactions. The two types of reversal procedure did not, therefore, impact significantly on the participants' mood or incentive rating measures.

\section{Discussion}

The current study showed the derived transfer of happy and sad mood functions through equivalence relations. Mood and incentive ratings recorded in the presence of the $B$ and $C$ stimuli that had been directly or indirectly related to happy music were higher than those for the $\mathrm{B}$ and $\mathrm{C}$ stimuli that were directly or indirectly related to sad music. These results 
lend firm support to the findings reported in the original study by BarnesHolmes et al. (2004).

One of the primary weaknesses noted in the original study (BarnesHolmes et al., 2004) was the absence of a baseline mood functions test to determine that the experimental stimuli had no preexperimentally established mood functions. The results from Stage 1 in the current study showed that the B stimuli to which the specific mood functions were subsequently attached, as well as the indirectly related $\mathrm{C}$ stimuli, did not produce specific mood functions prior to the experimental manipulations. One can conclude, therefore, that the subsequent changes in the mood functions of the stimuli were produced by the various experimental procedures.

As well as extending the original study by Barnes-Holmes et al. (2004), the current findings also support the existing literature that documents the transfer of functions through derived equivalence relations (Barnes \& Keenan, 1993; Barnes et al., 1995; Dougher et al., 1994; Dymond \& Barnes, 1994). Furthermore, the data support previous research on musical mood-induction procedures and their utility in generating mood functions (Clark \& Teasdale, 1985; Samson \& Rachman, 1989).

In addition to replicating clear transfer effects, the current work extended the Barnes-Holmes et al. (2004) study with the inclusion of two reversal procedures to demonstrate that the transfer effects, once established, were manipulable within the experimental setting. Within these reversal procedures, either the mood functions attached to the $B$ stimuli were altered (Reversal 1) or the classes in which the B stimuli participated were altered (Reversal 2). The results showed that both reversal procedures produced the predicted changes in mood functions.

A number of other studies in the behavioral literature have examined different types of equivalence reversal procedures. Researchers have identified two common types of reversal: "complete" and "partial" (e.g., Smeets, Barnes-Holmes, Akpinar, \& Barnes-Holmes, 2003). In a complete reversal, all of the stimulus relations established during the conditional discrimination training are reversed. These preparations have tended to produce reliable reversal of the derived equivalence relations (Spradlin, Cotter, \& Baxley, 1973; Wilson \& Hayes, 1996). In contrast, in a "partial" reversal, only some of the explicitly trained relations are reversed and there are conflicting findings with regard to its efficacy in reversing equivalence relations (Dube \& Mcllvane, 1995; Pilgrim, Chambers, \& Galizio, 1995).

In the current study, Reversal 1 simply involved switching the specific mood functions attached to the $B$ and $C$ stimuli but did not involve altering the conditional discriminations or equivalence relations in any way. As a result, it is neither a complete nor partial equivalence reversal as traditionally defined. Reversal 2 , however, may be referred to as a partial reversal, and the findings of the current study indicated reliable formation of the two new equivalence classes. In fact, all 8 participants demonstrated the new equivalence relations on their first exposure to the equivalence test after the reversal training (data not presented). These results differ 
from those reported by Pilgrim and Galizio $(1990,1995)$, who found that a partial reversal with two 3-member classes resulted in reversed symmetry but not reversed equivalence relations (see also Pilgrim et al., 1995). On balance, the present findings are consistent with those of other studies that have shown reliable reversals using the "partial" procedure (e.g., Smeets et al., 2003). At the present time, it remains unclear why this discrepancy in the equivalence literature has emerged, but the current data indicate once again that difficulty in reversing equivalence relations is far from ubiquitous.

The incentive ratings obtained from the current study were similar to those in the original study by Barnes-Holmes et al. (2004) in that they were all nonsignificant, although the effects were in the predicted direction. The incentive scale was adapted from Clarke and Teasdale (1985), who have reported statistically significant effects, but with larger numbers of participants (i.e., 64) than were used in the current study. It is possible, therefore, that the incentive rating scale simply constitutes a less sensitive (but not completely insensitive) measure of mood than the mood scale. That the incentive ratings were in the predicted direction in both the previous and current studies provides some support for the incentive scale as a measure of mood induction.

One possible limitation of the current and previous studies is the sole reliance on verbal self-reports as a measure of mood states. That is, perhaps participants' responses to the questionnaires did not reflect current mood but instead constituted a complex form of instructional control supported by the demand characteristics of the experiment. The susceptibility of self-report measures to extraneous variables is widely recognized, but alternative valid and reliable measures of mood states have been difficult to identify and many critical questions remain unanswered (e.g., de Raedt, Schacht, Franck, \& de Houwer, 2006). One way to control for demand is to use an instrument that is not immediately recognizable by the participant as relevant to the domain in question. In the case of the current study, the incentive scale was used for this purpose. As outlined above, although the incentive effects were not significant, they were in the predicted direction, they were consistent with the results of our previous study (Barnes-Holmes et al., 2004), and in any case the analyses were seriously underpowered relative to previous mood research with this scale. At present, therefore, it seems reasonable to conclude that the effects reported in the current study were not entirely due to demand and that the results did reflect at least some change in actual mood states.

The current and previous research (Barnes-Holmes et al., 2004) employed a well-established musical mood-induction procedure that is widely recognized to be a valid and reliable methodology (Clark, 1983; Martin, 1990). At the present time, it is not possible to determine whether the mood effects reported in the current study were largely due to the musical stimuli. Perhaps, consistent mood changes would have been observed if participants had simply been asked to think about happy 
or sad events for a few minutes without the music. Although previous studies indicate that the music does have a relatively powerful effect, future research on the derived transfer of mood functions could explore alternative methods for inducing mood states.

The current findings suggest that changing the psychological function of a stimulus may be achieved either through changing the function of the stimulus or related stimuli directly (Reversal 1 ) or through changing the derived relations in which the stimulus participates (Reversal 2). The results of the current study suggest that both approaches are equally effective in the context of a mood-induction procedure with a nonclinical population. It remains to be seen whether the same result is obtained with different experimental procedures or with the use of clinical populations, or both.

\section{References}

BARNES, D., BARNES, M., SMEETS, P. M., \& ROCHE, B. (1995). A transfer of functions and a conditioned transfer of functions through equivalence relations in three- to six-year-old children. The Psychological Record, 45, 405-430.

BARNES, D., \& KEENAN, M. (1993). A transfer of functions through derived arbitrary and non-arbitrary stimulus relations. Journal of the Experimental Analysis of Behavior, 59, 61-81.

BARNES-HOLMES, Y., BARNES-HOLMES, D., SMEETS, P. M., \& LUCIANO, C. (2004). The derived transfer of mood functions through equivalence relations. The Psychological Record, 54, 95-114.

BROWN, J., \& MANKOWSKI, T. (1993). Self-esteem, mood and self-evaluation: Changes in mood and the way you see you. Journal of Personality and Social Psychology, 3, 421-430.

CLARK, D. (1983). On the induction of the depressed mood in the laboratory: Evaluation and comparison of the Velten and musical procedures. Advances in Behavior and Therapy, 5, 27-49.

CLARK, D., \& TEASDALE, J. (1985). Constraints on the effects of mood on memory. Journal of Personality and Social Psychology, 48, 1595-1608.

DE RAEDT, R., SCHACHT, R., FRANCK, E., \& DE HOUWER, J. (2006). Selfesteem and depression revisited: Implicit positive self-esteem in depressed patients? Behaviour Research and Therapy, 44, 1017-1028.

DOUGHER, M. J., AUGUSTON, E., MARKHAM, M. R., GREENWAY, D., \& WULFERT, E. (1994). The transfer of respondent eliciting and extinction through equivalence classes. Journal of the Experimental Analysis of Behavior, 62, 331-351.

DUBE, W. W., \& MCILVANE, W. J. (1995). Stimulus-reinforcer relations and emergent matching to sample. The Psychological Record, 45, 591-612.

DYMOND, S., \& BARNES, D. (1994). A transfer of self-discrimination response functions through equivalence relations. Journal of the Experimental Analysis of Behavior, 62, 251-267.

HAYES, S. C., BARNES-HOLMES, D., \& ROCHE, B. (Eds.). (2001). Relational frame theory: A post-Skinnerian account of human language and cognition. New York: Plenum. 
HAYES, S. C., FOX, E., GIFFORD, E. V, WILSON, K. G., BARNES-HOLMES, D., \& HEALY, O. 2001). Derived relational responding as learned behavior. In S. C. Hayes, D. Barnes-Holmes, \& B. Roche (Eds.), Relational frame theory: A post-Skinnerian account of human language and cognition (pp. 21-51). New York: Plenum.

MARTIN, M. (1990). On the induction of mood. Clinical Psychology Review, 10, 139-697.

PILGRIM, C., CHAMBERS, L., \& GALIZIO, M. (1995). Reversal of baseline relations and stimulus equivalence: II. Children. Journal of the Experimental Analysis of Behavior, 63, 239-254.

PILGRIM, C., \& GALIZIO, M. (1990). Relations between baseline contingencies and equivalence probe performances. Journal of the Experimental Analysis of Behavior, 54, 213-224.

PILGRIM, C., \& GALIZIO, M. (1995). Reversal of baseline relations and stimulus equivalence: I. Adults. Journal of the Experimental Analysis of Behavior, 63, 225-238.

ROCHE, B., \& BARNES, D. (1997). A transformation of respondently conditioned stimulus function in accordance with arbitrarily applicable relations. Journal of the Experimental Analysis of Behavior, 67, 275-300.

SAMSON, D., \& RACHMAN, S. (1989). The effect of induced mood on fear reduction. British Journal of Clinical Psychology, 28, 227-238.

SIDMAN, M. (1971). Reading and auditory-visual equivalences. Journal of Speech and Hearing Research, 14, 5-13.

SIDMAN, M. (1994). Equivalence relations and behavior: A research story. Boston: Authors Cooperative.

SMEETS, P. M., BARNES-HOLMES, Y., AKPINAR, D., \& BARNES-HOLMES, D. (2003). Reversal of equivalence relations. The Psychological Record, 53, 91-120.

SPRADLIN, J. E., COTTER, V. W., \& BAXLEY, N. (1973). Establishing a conditional discrimination without direct training: A study of transfer with retarded adolescents. American Journal of Mental Deficiency, 77, 556566.

TEASDALE, J. D., \& SPENCER, P. (1982). Effects of induction elation and depression on estimates of past success and subjective probability of future success. Unpublished manuscript, University of Oxford, England.

WILSON, K. G., \& HAYES, S. C. (1996). Resurgence of derived stimulus relations. Journal of the Experimental Analysis of Behavior, 66, 267-281. 